\title{
Control of leg movements driven by EMG activity of shoulder muscles
}

\section{Valentina La Scaleia ${ }^{1,2}$ *, Francesca Sylos-Labini ${ }^{1,2}$, Thomas Hoellinger ${ }^{3}$, Letian Wang ${ }^{4}$, Guy Cheron ${ }^{3}$, Francesco Lacquaniti ${ }^{1,2,5}$ and Yuri P. Ivanenko ${ }^{1}$}

1 Laboratory of Neuromotor Physiology, Santa Lucia Foundation, Rome, Italy

${ }^{2}$ Centre of Space Bio-Medicine, University of Rome Tor Vergata, Rome, Italy

${ }^{3}$ Laboratory of Neurophysiology and Movement Biomechanics, Université Libre de Bruxelles, Brussels, Belgium

${ }^{4}$ Department of Biomechanical Engineering, University of Twente, Enschede, Netherlands

${ }^{5}$ Department of Systems Medicine, University of Rome Tor Vergata, Rome, Italy

\section{Edited by:}

Ana Bengoetxea, Universidad del País Vasco-Euskal Herriko Unibertsitatea (UPV/EHU), Spain

\section{Reviewed by:}

Vincent C. K. Cheung, Massachusetts Institute of Technology, USA

Tomoyoshi Komiyama, Chiba

University, Japan

\section{*Correspondence}

Valentina La Scaleia, Laboratory of Neuromotor Physiology, IRCCS Fondazione Santa Lucia, 306 via Ardeatina, Rome 00179, Italy e-mail: v.lascaleia@hsantalucia.it
During human walking, there exists a functional neural coupling between arms and legs, and between cervical and lumbosacral pattern generators. Here, we present a novel approach for associating the electromyographic (EMG) activity from upper limb muscles with leg kinematics. Our methodology takes advantage of the high involvement of shoulder muscles in most locomotor-related movements and of the natural co-ordination between arms and legs. Nine healthy subjects were asked to walk at different constant and variable speeds (3-5 km/h), while EMG activity of shoulder (deltoid) muscles and the kinematics of walking were recorded. To ensure a high level of EMG activity in deltoid, the subjects performed slightly larger arm swinging than they usually do. The temporal structure of the burst-like EMG activity was used to predict the spatiotemporal kinematic pattern of the forthcoming step. A comparison of actual and predicted stride leg kinematics showed a high degree of correspondence $(r>0.9)$. This algorithm has been also implemented in pilot experiments for controlling avatar walking in a virtual reality setup and an exoskeleton during over-ground stepping. The proposed approach may have important implications for the design of human-machine interfaces and neuroprosthetic technologies such as those of assistive lower limb exoskeletons.

Keywords: arm-leg co-ordination, quadrupedal locomotion, EMG patterns, gait kinematics, neuroprosthetic technology

\section{INTRODUCTION}

Neuroprosthetic devices based on brain-machine interface (BMI) technology have the potential to restore mobility to both upper and lower extremities and to enable walking (Millán et al., 2010; Lebedev et al., 2011; Cheron et al., 2012; Wolpaw, 2013). Exoskeleton robotic devices are extensively developed in recent years to provide new possibilities for severely paralyzed patients to walk (Sale et al., 2012; Malcolm et al., 2013; Wang et al., 2013; Sylos-Labini et al., 2014b). The development of practical applications for gait assistance may involve a wide range of potential control implementations, from using trunk movements (Wang et al., 2013) or myoelectric signals (del-Ama et al., 2012; Alcaide-Aguirre et al., 2013; Gordon et al., 2013) to implementing BMI-based technology (Fitzsimmons et al., 2009; Gwin et al., 2011). To develop effective neuroprosthetic devices for human beings, BMI research has to address a number of issues related to improving the quality of neuronal recordings, and extending the BMI approach to a broad range of motor and sensory functions (Lebedev et al., 2011). In human beings, non-invasive electroencephalogram-based brain-machine interfacing for providing a reliable control of walking in the exoskeleton is still very limited (Gwin et al., 2011; Cheron et al., 2012; Wagner et al., 2012), and alternative or supplementary control strategies should be considered in parallel. The current study investigates a novel approach for associating the upper limb electromyographic (EMG) activity with leg kinematics. This approach holds promise for applications in the field of assistive lower limb exoskeletons.

The co-ordination of limb and body segments in locomotion arises from stereotyped coupling of cervical and lumbosacral spinal segment outputs (Ballesteros et al., 1965; Murray et al., 1967; Hogue, 1969; Wannier et al., 2001; Zehr and Duysens, 2004; Ivanenko et al., 2006; De Sèze et al., 2008; Barthelemy and Nielsen, 2010; Meyns et al., 2013; Sylos-Labini et al., 2014a). The coordination between arms and legs during human locomotion shares many features with that in quadrupeds (Falgairolle et al., 2006; Patrick et al., 2009; Juvin et al., 2012; MacLellan et al., 2012). Due to the natural arm-leg co-ordination in human walking, the leg movement control can be derived from or reflected in the EMGs of arm muscles. In particular, a group of the upper arm muscles was shown to be active during many locomotion tasks in human beings (Ivanenko et al., 2006; Kuhtz-Buschbeck and Jing, 2012). Taking into account, the high involvement of the deltoid muscle in most locomotor-related movements in human beings, we took advantage of the natural arm-leg co-ordination for investigating the control of stepping using the upper limb EMG activity. Here, 
we tested the hypothesis that the temporal structure of EMG activity of shoulder muscles can be used to predict the spatiotemporal kinematic pattern of the forthcoming step during actual gait at 3$5 \mathrm{~km} / \mathrm{h}$ (and we investigated the accuracy of this prediction) and to control avatar walking in a virtual reality setup or an exoskeleton during over-ground stepping.

\section{MATERIALS AND METHODS PARTICIPANTS AND PROTOCOLS}

Two main protocols were implemented. In the first series of experiments performed in Brussels (protocol 1), we investigated the possibility of extracting lower limb kinematic data from the gaitrelated rhythmic EMG activity of arm muscles. To this end, nine healthy volunteers were enrolled [age range 23-33 years, four males and five females, leg length $0.85 \pm 0.04 \mathrm{~m}$ (mean $\pm \mathrm{SD}$ ), height $1.75 \pm 0.10 \mathrm{~m}$, weight $72 \pm 7 \mathrm{~kg}$ ]. In the second series of experiments performed in Rome (protocol 2), we implemented the on-line control of avatar walking and also over-ground walking in the exoskeleton. For this protocol, eight healthy volunteers were enrolled (age range 25-53 years, five males and three females, leg length $0.83 \pm 0.04 \mathrm{~m}$, height $1.77 \pm 0.09 \mathrm{~m}$, weight $69 \pm 8 \mathrm{~kg}$ ). None of the subjects had any known neurological or motor disorder. These protocols were approved by the Ethics Committees of the Université Libre de Bruxelles (Belgium) and Fondazione Santa Lucia (Rome, Italy), respectively, and all subjects gave their informed written consent prior to participation.

\section{EXPERIMENTAL SETUP AND DATA ANALYSIS \\ Protocol 1}

In the first protocol, the subjects were asked to walk on a treadmill (Cosmed Treadmill T150) at different constant speeds (3, 4, and $5 \mathrm{~km} / \mathrm{h}$ ) and at a variable speed (ramp-and-hold velocity profile, increasing from 3 to $5 \mathrm{~km} / \mathrm{h}$ at $0.1 \mathrm{~km} / \mathrm{h} / \mathrm{s}$ ), four trials total ( 3 constant speeds +1 trial at a variable speed). On average, $10-15$ strides were recorded in each trial during walking at a constant speed (that corresponds to a walking distance of $\sim 15-20 \mathrm{~m}$ ) and about 50 strides during walking at a variable speed (distance $\sim 70 \mathrm{~m}$ ). During normal walking, the amplitude of arm swinging depends on the walking speed (Webb et al., 1994) and, at slow speeds, the activity of arm muscles decreases significantly (Ivanenko et al., 2006; Kuhtz-Buschbeck and Jing, 2012). Therefore, to obtain a higher level of arm EMG activity, the subjects were asked to perform slightly larger arm swinging than they usually do. In the preliminary training sessions (2-3 min of walking), we verified that these movements evoked consistent EMG activation of shoulder muscles at all walking speeds $(3-5 \mathrm{~km} / \mathrm{h})$. We recorded kinematic data bilaterally at $100 \mathrm{~Hz}$ by means of the Vicon system (Vicon, Oxford, $\mathrm{UK}$ ) with 10 Bonita cameras spaced around the treadmill. Infrared reflective markers (diameter $15 \mathrm{~mm}$ ) were attached on each side of the subjects to the skin (using Vicon BioMind asymmetric model), in particular, overlying the following landmarks used to calculate leg kinematics: greater trochanter (GT), lateral femur epicondyle (LE), lateral malleolus (LM), and fifth metatarsophalangeal joint (VM). EMG activity of the anterior deltoid (DELTa) and posterior deltoid (DELTp) muscles was recorded bilaterally by means of surface electrodes. The EMG data were recorded with the wireless BTS Freeemg system (BTS Bioengineering, Milano, Italy) and digitized at $1000 \mathrm{~Hz}$. Sampling of kinematic and EMG data were synchronized.

\section{Data processing}

Our approach uses the timing of the burst-like EMG activity of shoulder muscles (by applying the peak detection algorithm) to predict the spatiotemporal kinematic pattern of the forthcoming step. Prior to application of the peak detection algorithm, the EMG data were pre-processed: high-pass filtered at $30 \mathrm{~Hz}$, rectified, and finally low-pass filtered (all filters, zero-lag fourth order Butterworth). Low-pass filtering was performed at different frequencies $(1 \div 5 \mathrm{~Hz})$ to achieve the best correlation between actual and predicted leg kinematics. Despite some inter-individual variability, periods of EMG activity of DELTa and DELTp during normal walking tend to be alternating and correspond to those of the contralateral upper limb (Ivanenko et al., 2006; Kuhtz-Buschbeck and Jing, 2012), as well as multi-muscle synergy-based control interface may be more efficient than a single-muscle control (Lunardini et al., 2014). Therefore, bilateral EMGs of synergistic muscles (Ivanenko et al., 2006; Kuhtz-Buschbeck and Jing, 2012) were summed (Figure 1A):

$$
\begin{aligned}
& \mathrm{EMG}_{1}=\mathrm{DELTa}_{\text {right }}+\text { DELTp }_{\text {left }} \\
& \mathrm{EMG}_{2}=\mathrm{DELTa}_{\text {left }}+\text { DELTp }_{\text {right }}
\end{aligned}
$$

the peaks of $\mathrm{EMG}_{1}$ and $\mathrm{EMG}_{2}$ occur around the beginning of the swing phase of the right and left legs, respectively (Figure 1A). Nevertheless, we also compared the performance of our algorithm using all four muscles (Eqs 1 and 2) and only pairs of contralateral

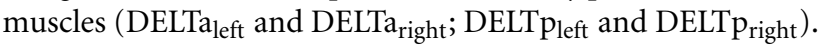

The elevation angles of the thigh (GT-LE), shank (LE-LM), and foot (LM-VM) segments (Lacquaniti et al., 2002; Cheron et al., 2012) were calculated as the angles between the segment projected on the sagittal plane and the vertical (positive in the forward direction, i.e., when the distal marker falls anterior to the proximal one). We divided the recorded kinematic and EMG data into gait cycles (maximum shank elevation angle as the beginning of the gait cycle), then interpolated each stride to 200 time points, and finally averaged across gait cycles (for each trial). These ensembleaveraged (thigh, shank, and foot) elevation angles were used as a reference (template) for predicting leg kinematics from shoulder muscle EMGs. The amplitude of arm oscillations was assessed as the peak-to-peak amplitude of anterior-posterior wrist marker movements relative to the shoulder averaged across both (left and right) sides of the body and across all strides of the trial.

\section{Prediction of leg kinematics based on shoulder muscle EMG activity}

The algorithm used to predict the kinematics of the following step from the temporal structure of pre-processed EMG activity is schematically depicted in Figure 1. In essence, it predicts stride duration from the temporal distance between EMG peaks, and the kinematic pattern of a reference template (derived from averages of the collected data) is then temporally stretched to fit the predicted duration so as to generate the predicted segment elevation angles. The algorithm started from the second recorded step and consisted in detecting the timing of the $\mathrm{EMG}_{1}$ or $\mathrm{EMG}_{2}$ peak (exceeding the pre-defined threshold) in the appropriate time 


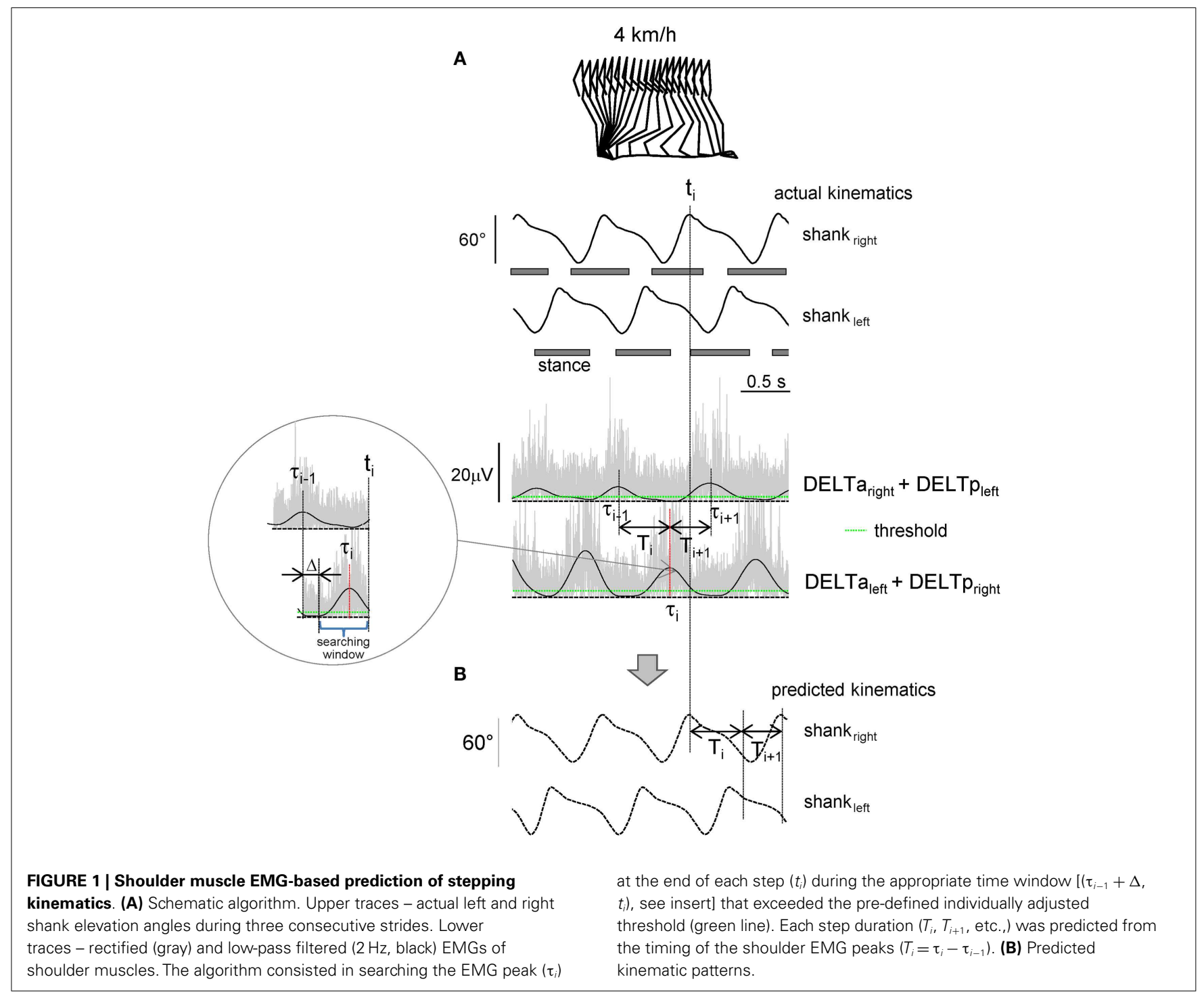

window and associating it with the duration and kinematics of the following step. In particular, at the end of each predicted step $\left(t_{i}\right)$, the program computed the timing of the latest peak of $\mathrm{EMG}_{1}$ or $\mathrm{EMG}_{2}$ depending on the associated time lag $\left(\tau_{i}\right.$, Figure 1A) and determined the duration of the following step:

$$
\begin{aligned}
T_{i} & =\tau_{i}-\tau_{i-1} \\
T_{i+1} & =\tau_{i+1}-\tau_{i} \\
& \ldots
\end{aligned}
$$

The peak detection threshold was defined individually for each trial and each EMG as

$$
\text { threshold }=k \times \text { mean }(\mathrm{EMG})
$$

where mean $(\mathrm{EMG})$ is the mean $\left(\mathrm{EMG}_{1}\right)$ or mean $\left(\mathrm{EMG}_{2}\right)$ over the trial, and $k$ is the coefficient found empirically $(k=0.8)$ to provide the best detection of peaks.
The searching window started with a fixed delay $\Delta=200 \mathrm{~ms}$ from the last EMG peak (Figure 1A) in order not to capture extra peaks (from the previous step). The kinematic pattern of the forthcoming step was calculated by scaling (stretching) the kinematic reference template (see above) according to the predicted $T_{i}$. The flexibility in the timing of the EMG peak due to accelerating/decelerating arm movements allowed gradual changes in the predicted cycle duration. On the other hand, in the case of the absence of the detected EMG peak at the time $t_{i}$, the algorithm did not produce further steps and was terminated (in such case the trial was not considered to be successful for the prediction of leg kinematics).

\section{Protocol 2}

In the second protocol, we implemented the on-line control of leg kinematics based on rhythmic activity of shoulder muscles in the virtual reality setup (eight subjects) and during walking in the exoskeleton (one subject). EMG activity of DELTa and DELTp muscles on each side was registered at $500 \mathrm{~Hz}$ by means of surface 
electrodes with the wireless Delsys Trigno EMG system (Delsys Inc., Boston, MA, USA), bandwidth of $20-450 \mathrm{~Hz}$, and overall gain of 1000. LabView software was used to collect and process the EMG data and to predict leg kinematics in real time. The predicted kinematic signals (thigh, shank, and foot elevation angles) were sent at $100 \mathrm{~Hz}$ using the UDP protocol to the virtual environment (using XVR software of VRMedia S.r.l.) to animate walking avatar (third person viewpoint). The algorithm was similar (Figure 1, the lowpass filter was performed at $2 \mathrm{~Hz}$, based on the results of the first protocol, see Results) although, in addition to the on-line step-bystep control of walking kinematics, it also included gait initiation and gait termination steps. The threshold for EMG peak detection (Eq. 4) was adjusted individually for each subject, computing the mean EMG activity during alternating arm movements at a selfselected rhythm in the preliminary trial. The subjects (in standing position) were instructed to swing their arms at approximately the same amplitude at self-selected, slow, and fast frequency, as well as at a variable frequency. We instructed the subjects to vary the frequency approximately in the range between the slow and fast frequencies performed in the previous trials. The duration of each trial was $1 \mathrm{~min}$ and three trials were performed at each arm frequency.

In a pilot experiment, one subject was also trained to control an exoskeleton during over-ground stepping along an 8-m walkway. The detailed description of the exoskeleton (called MINDWALKER, https://www.mindwalker-project.eu) is provided elsewhere (Wang et al., 2013). Briefly, knee and hip exoskeleton joints (powered by series elastic actuators) followed pre-defined joint angles (based on the walking patterns of the same subject walking in the MINDWALKER exoskeleton without assistance) provided with variable joint impedances (Wang et al., 2013). The algorithm was similar to that used in the first protocol and detected the timing of EMG peaks. The output of the LabView software triggered and determined the initiation and the duration of the swing phase of each leg.

\section{STATISTICS}

Descriptive statistics included means \pm SD of the mean. The efficiency and accuracy of the predicting algorithm during walking at a constant speed $(3,4$ and $5 \mathrm{~km} / \mathrm{h})$ were assessed using two parameters: the number of successful trials (if 10 consecutive strides were successfully predicted) and correlation between predicted and actual segment elevation angles. The efficiency of the method during walking at a variable speed $(3-5 \mathrm{~km} / \mathrm{h})$ was assessed using the correlation between predicted and actual segment elevation angles, and the correlation between predicted and actual stride durations. In protocol 2, since the actual leg kinematics was not performed, the efficiency of the on-line algorithm using a virtual reality setup was assessed by the percentage of successful trials (if the algorithm produced alternating uninterrupted steps during the 1-min trial) during slow, self-selected, and fast arm movements. Statistics on Pearson's correlation coefficients was performed on the normally distributed, $Z$-transformed values.

A repeated measure (RM) ANOVA was used to evaluate prediction (correlation coefficients between predicted and actual limb segment elevation angles), and post hoc Tukey's HSD test was used to determine statistical significance. In one subject, the algorithm failed to predict limb kinematics (presumably due to low-EMG activity, see Results) and his data were not included. In another subject, it failed at a high speed $(5 \mathrm{~km} / \mathrm{h})$ and the missing data for this condition for the ANOVA were replaced by the unweighted mean value estimated from all other subjects. Reported results are considered significant for $p<0.05$.

\section{RESULTS}

\section{SHOULDER MUSCLE ACTIVITY DURING WALKING}

The shoulder muscles we monitored (bilateral anterior and posterior deltoid) showed rhythmical EMG signals during walking in all subjects (on average, the amplitude of the main rectified EMG peak was $7.0 \pm 1.7 \mu \mathrm{V}$, all muscles and all speed being pooled together), although in a few cases their activity was small $(<3 \mu \mathrm{V})$. Since the subjects were asked to perform approximately the same amplitude of reciprocal arm swinging [forward arm swing reverses to backward arm swing in the middle of the gait cycle (Kuhtz-Buschbeck and Jing, 2012)], EMG activity did not decrease with decreasing walking speed, as it normally occurs during walking (Ivanenko et al., 2006). On average, the anterior-posterior arm (wrist marker) oscillations were $50 \pm 12 \mathrm{~cm}$ at $3 \mathrm{~km} / \mathrm{h}, 45 \pm 9 \mathrm{~cm}$ at $4 \mathrm{~km} / \mathrm{h}$, and $41 \pm 10 \mathrm{~cm}$ at $5 \mathrm{~km} / \mathrm{h}$ (for instance, during normal walking at 4$5 \mathrm{~km} / \mathrm{h}$, arm oscillations are $\sim 35-40 \mathrm{~cm}$, Murray et al., 1967; Webb et al., 1994; Ford et al., 2007), and the mean amplitude of deltoid muscle EMGs was $7.6 \pm 1.7 \mu \mathrm{V}$ at $3 \mathrm{~km} / \mathrm{h}, 7.0 \pm 1.7 \mu \mathrm{V}$ at $4 \mathrm{~km} / \mathrm{h}$, and $6.1 \pm 1.3 \mu \mathrm{V}$ at $5 \mathrm{~km} / \mathrm{h}$.

Figure 2A illustrates an example of shoulder muscle EMG signals during walking at $4 \mathrm{~km} / \mathrm{h}$. Typically, the deltoid muscle demonstrated alternating activity during walking: alternation occurred both between left and right sides of the body and between anterior and posterior bellies of the deltoid (DELTa and DELTp). However, there could be an additional smaller second burst of activity over the gait cycle, as well as some inter-individual variability in the timing of the main EMG bursts [see also Ballesteros et al. (1965), Hogue (1969), Ivanenko et al. (2006), Kuhtz-Buschbeck and Jing (2012)]. Nevertheless, in most cases, there were prominent peaks of $\mathrm{EMG}_{1}$ and $\mathrm{EMG}_{2}$ around the beginning of the swing phase of the right and left legs, respectively (Figures 1A and 2A), which allowed us to associate this phasic alternating pattern of the upper limb EMG activity during arm-leg co-ordination with the spatiotemporal pattern of gait kinematics.

\section{PREDICTING LEG KINEMATICS FROM SHOULDER MUSCLE EMGs DURING WALKING}

We used the rectified and filtered EMG data to detect the timing of shoulder muscle EMG peaks in the concomitant and previous steps (see Materials and Methods) to predict the limb kinematics in the forthcoming step. On the whole, the best correlation between actual and predicted kinematics was observed at $1-2 \mathrm{~Hz}$ low-pass filtering $(r>0.95$, Figure 2B), with slightly lower percentage of successful trials at $1 \mathrm{~Hz}$ low-pass at faster walking speeds (Figure 2C, presumably because the cut-off frequency was close to the step cycle frequency and the algorithm failed to detect EMG peak in time and was terminated), so that in the following we will present the results obtained at $2 \mathrm{~Hz}$ low-pass.

The percentage of subjects with a successful prediction of consecutive strides (2 Hz low-pass) from shoulder EMGs activity is 

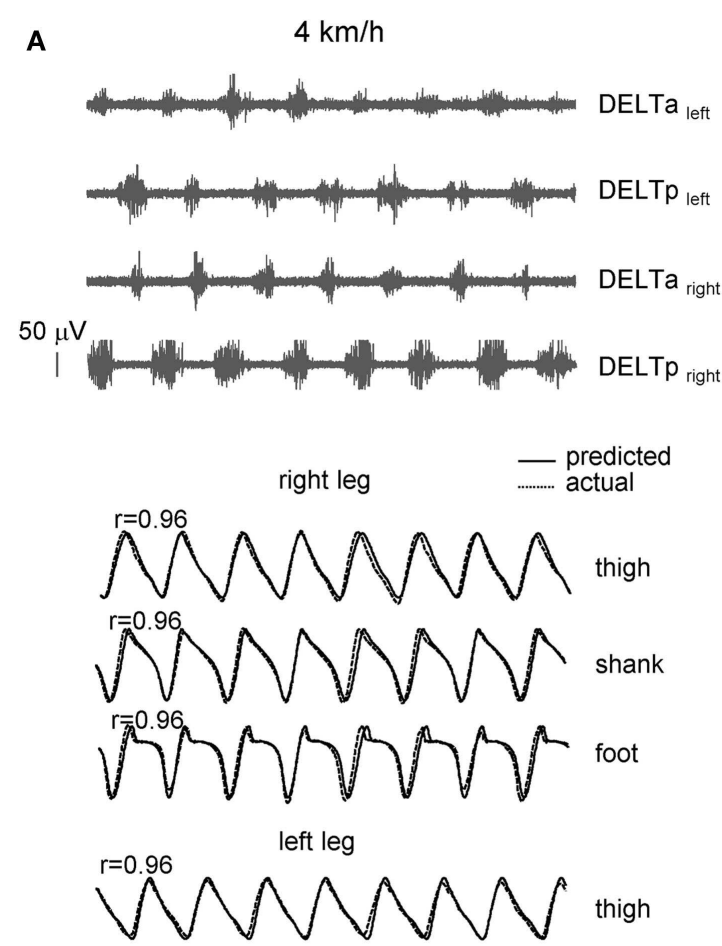
$\sqrt{r=0} \mathrm{~N} N \mathrm{~N} N \mathrm{~N} /$ shank
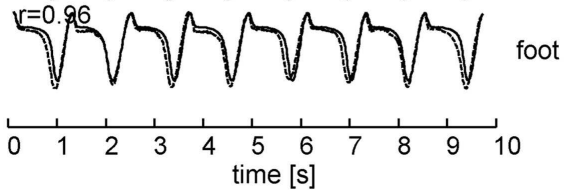

FIGURE 2 | Performance of leg kinematics prediction algorithm using shoulder muscle EMGs during walking at constant speeds. (A) An example of muscle activity and kinematic patterns of one subject during walking at $4 \mathrm{~km} / \mathrm{h}$. Note, a fairly good correspondence between predicted (solid lines) and actual (dotted lines) thigh, shank, and foot elevation angles. (B) Correlation coefficients (averaged across all steps and subjects) between predicted and real shank segment elevation

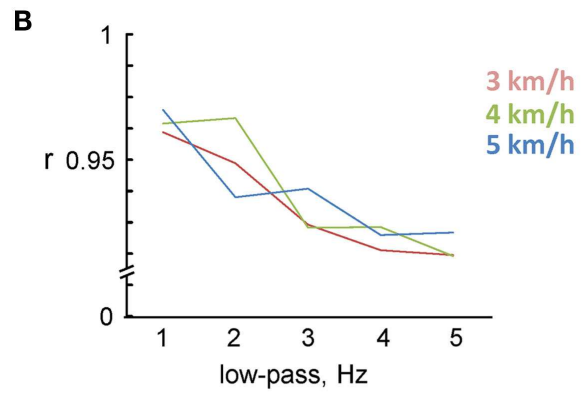

C

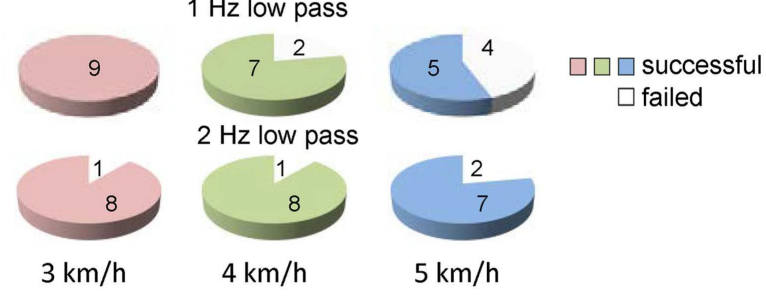

D

correlation between actual and predicted leg kinematics

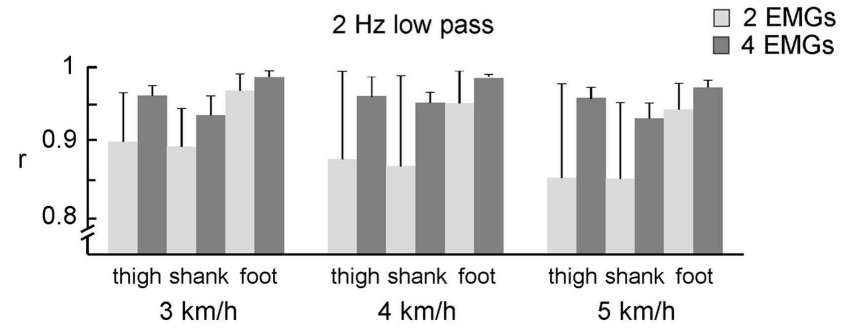

angles using different cut-off frequencies of low-pass filter. (C) Pie charts showing the percentage of subjects with a successful 10 consecutive strides prediction from shoulder EMGs activity (both 1 and $2 \mathrm{~Hz}$ low-pass filter for each speed). (D) Correlation ( $+S D$ ) between actual and predicted kinematic patterns of individual subjects (2 Hz low-pass) using two and four shoulder muscle EMGs. Note, better predictions when using four EMGs. shown in Figure 2C (lower plots). In one subject, EMG activity was rather low and the algorithm failed to predict gait kinematics. Some variability in EMG amplitude is to be expected due to individual differences in skin impedance and/or individual differences in the upper limb muscle EMG patterns during walking (KuhtzBuschbeck and Jing, 2012). Nevertheless, in most subjects (eight out of nine), it was successful at all speeds of walking.

In addition to the high rate of prediction (Figure 2C), the correlation between predicted and actual leg kinematics in successful trials was also high (Figure 2D). Figure 2A (lower curves) illustrates an example of actual (dotted lines) and predicted (solid lines) leg segment elevation angles during walking at $4 \mathrm{~km} / \mathrm{h}$. Despite some discrepancies (small phase shifts in the kinematic patterns of individual steps), there was a fairly good correspondence to actual lower limb kinematics, and a 1:1 ratio between frequencies of predicted and actual steps (absence of step "doubling" and absence of missing steps). The high-correlation coefficients between actual and predicted limb segment elevation angles $(r>0.9$, Figure 2D) suggest stability of phasic alternating pattern of the upper limb EMG activity across consecutive strides.

We compared the performance of our algorithm using all four muscles (Eqs 1 and 2) and only two contralateral muscles $\left(\right.$ DELTa $_{\text {left }}$ and DELTa ${ }_{\text {right }} ;$ DELTp $_{\text {left }}$ and DELTp right $)$. In the latter case, correlation coefficients of two pairs of muscles were averaged, since in $\sim 30 \%$ of trials only one pair of contralateral muscles could predict leg kinematics while another pair failed to do it due to the relatively low/variable activity. The RM ANOVA (2 number of muscles $\times 3$ speeds $\times 3$ segments) resulted in a significant effect for muscle $[F(1,7)=9.686, p=0.017]$, segment $[F(2,14)=34.18$, $p<0.00001]$ and muscle $\times$ segment interaction $[F(2,14)=9.888$, $p=0.002]$. Post hoc tests revealed that correlations were higher when using four EMGs and they were also higher for the foot 
elevation angle relative to the shank and thigh elevation angles (Figure 2D). Thus, both the percentage of successful trials and the correlation coefficients were higher when using four shoulder muscles for prediction compared with only two muscles.

We also investigated the performance of the proposed leg kinematics prediction algorithm during walking at a variable speed $(3-5 \mathrm{~km} / \mathrm{h})$. Figure 3B shows an example of cycle durations of all individual strides in one subject (left panel) and all successful $(n=8)$ subjects (right panel). The actual stride duration varied between 0.85 and $1.43 \mathrm{~s}$, while the predicted stride duration also varied in a similar though slightly larger range (between 0.7 and $1.55 \mathrm{~s}$, Figure 3B). The correlation between the reference template and individual cycles used for computing the template was high (on average $0.98 \pm 0.01$, the data for all segments were pooled together) consistent with a relatively low inter-stride variability of the segment elevation angles (Borghese et al., 1996; Bianchi et al., 1998). Therefore, inter-stride variability in the predicted stride durations and leg kinematics is likely related (at least in part) to inter-stride variability in the EMG patterns that has been documented for both leg and arm muscle activity during human walking (Kang and Dingwell, 2009; Kuhtz-Buschbeck and Jing, 2012; Zelik et al., 2014). On the whole, the relationship between predicted and actual stride duration was linear (Figure 3B) and correlation between predicted and actual leg kinematics was high $(r>0.95$, Figure 3C). RM ANOVA resulted in a significant effect for segment $[F(2,14)=7.789, p=0.0053]$ and post hoc tests revealed higher correlations for the foot elevation angle relative to the shank segment $(p=0.0045)$.

\section{CONTROLLING VIRTUAL AVATAR AND EXOSKELETON}

The suggested algorithm has also been implemented in the pilot experiments to trigger steps and control avatar walking (Figure 4A) and an exoskeleton during over-ground stepping (Figure 4B). Each trial consisted of the three locomotor-related phases controlled by the timing of EMG peaks: gait initiation, walking at a variable speed, and gait termination. In the absence of the EMG peak, the virtual avatar or exoskeleton did not produce further steps and gait termination was performed. The exoskeleton was tested only in one trained subject since typically the wearer has to use crutches to guarantee lateral stability (Wang et al., 2013).

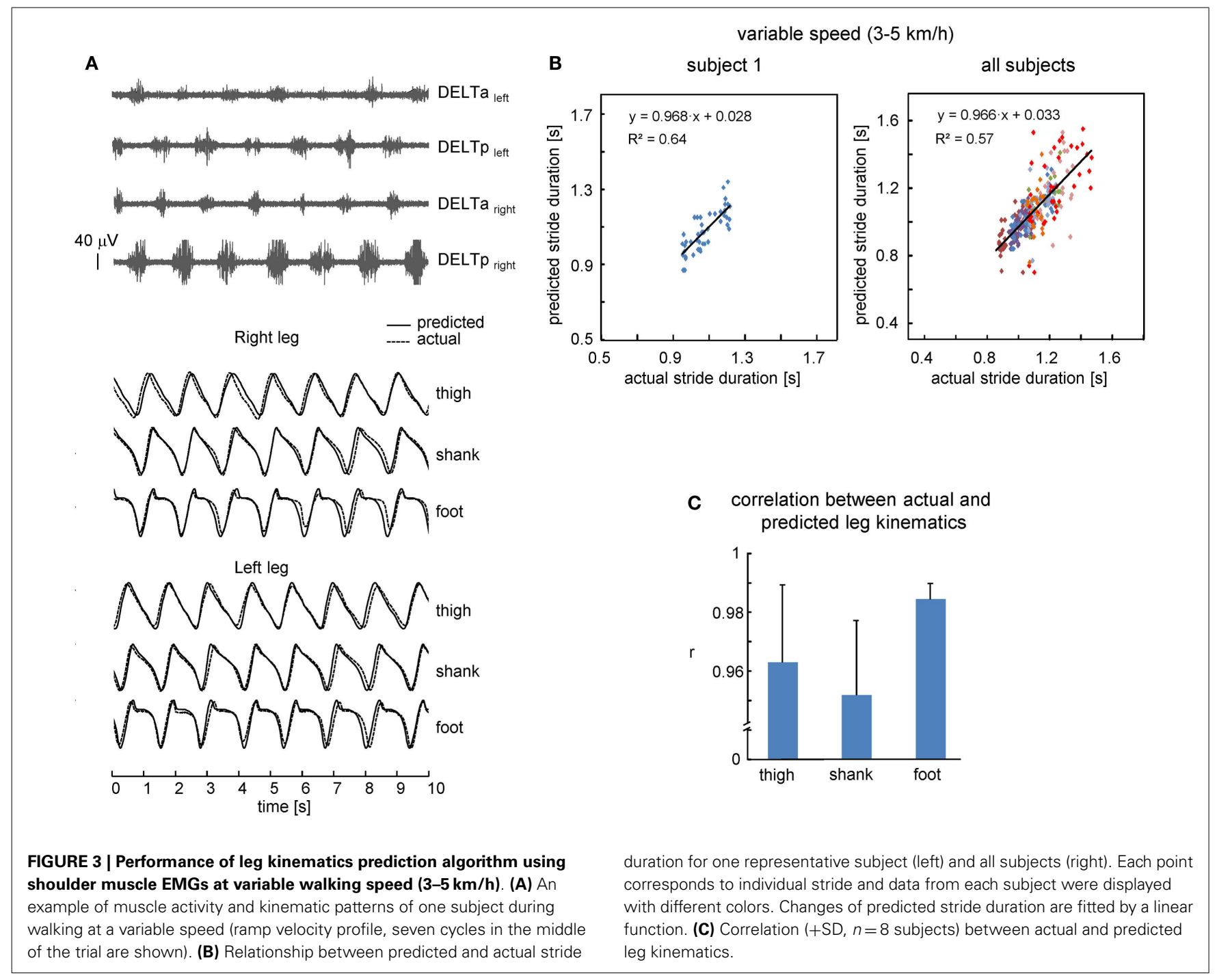


A
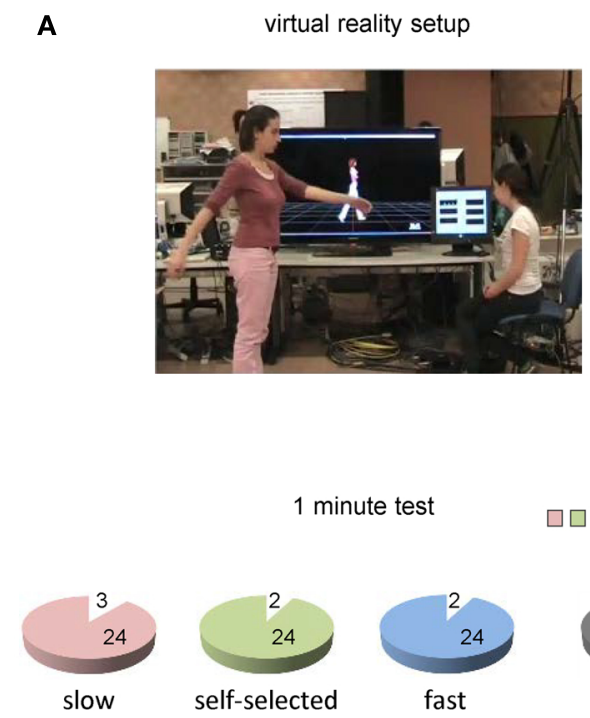

self-selected

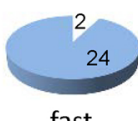

fast $\square \square \square \square$ successful $\square$ failed

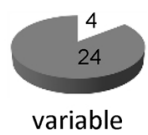

FIGURE 4 | On-line shoulder muscle EMG control of leg movements (A) Controlling of walking avatar in a virtual reality setup (third person viewpoint). To control the timing and duration of individual steps, the subject produced alternating arm swinging movements in standing position (upper panel). Lower panel - pie charts showing the percentage of trials with a successful 1-min test for producing stepping (if the algorithm predicted consecutive uninterrupted steps during the 1-min trial) using alternating arm
B
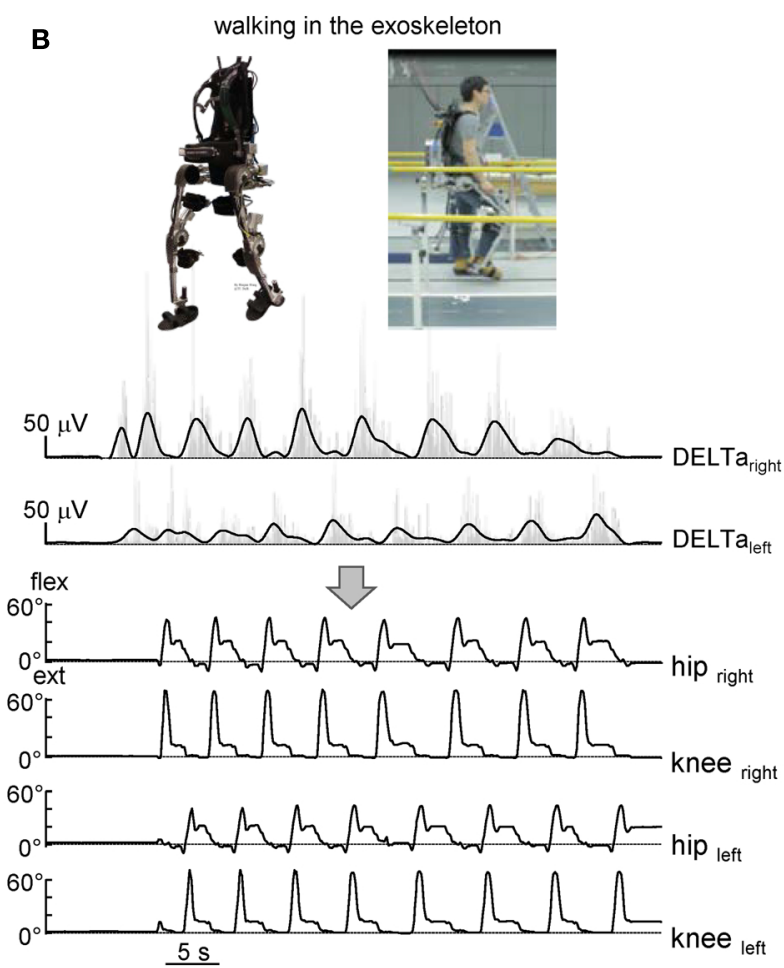

swinging at different frequencies ( $n=8$ subjects, 24 trials total for each condition). (B) Arm EMG-based control of stepping in the exoskeleton by the healthy subject. Upper traces - rectified (gray) and low-pass filtered (black) EMGs of shoulder muscles. Each step duration and initiation were calculated and triggered based on the timing of the shoulder EMG peaks. Bottom traces - knee and hip joint angle kinematic patterns of eight consecutive steps along a 9-m walkway.
Nevertheless, this subject succeeded to use alternating EMG bursts of shoulder muscles to trigger 7-10 consecutive strides along a 8-m walkway (Figure 4B). Again, the percentage of successful trials for controlling virtual avatar at slow, self-selected, and fast frequency of arm movements was high (Figure 4A, lower panel) and similar to that found in the first experiment (Figure 2C) even though different subjects participated in the two protocols.

\section{DISCUSSION \\ ARM SWINGING DURING LOCOMOTION}

Arm swing during human walking is not an entirely passive movement, as also shown by the observation that upper limb muscles show rhythmic activity contributing to arm swing (Ballesteros et al., 1965; Hogue, 1969; Ivanenko et al., 2006). Interestingly, rhythmic muscle activity continues to some extent even when the arm is immobilized (Kuhtz-Buschbeck and Jing, 2012). During walking, the EMG activity associated with arm swing is feeble compared with the EMG activity during maximum voluntary contractions. For instance, the mean amplitude values depend on speed and are lower at $4 \mathrm{~km} / \mathrm{h}$ than at $6 \mathrm{~km} / \mathrm{h}$ (Kuhtz-Buschbeck and Jing, 2012). However, even during fast walking $(6 \mathrm{~km} / \mathrm{h})$, they remain well below $5 \%$ of maximum voluntary contraction, and yet, EMG patterns show prominent peaks around specific phases of the gait cycle. During running $(7-12 \mathrm{~km} / \mathrm{h})$, the activity of shoulder muscles increases $\sim 2-3$ times relative to walking at $5 \mathrm{~km} / \mathrm{h}$ (Cappellini et al., 2006) and also demonstrates a strong coupling between cervical and lumbosacral spinal motoneuron output (Ivanenko et al., 2008).

Previous studies describe this behavior both as a biomechanical advantage (Ortega et al., 2008; Park, 2008; Collins et al., 2009; Meyns et al., 2013) and a neural/evolutionary mechanism based on propriospinal pathways connecting distinct spinal segments, as seen in invertebrates and quadrupedal mammals (Falgairolle et al., 2006). Moreover, many features of quadrupedal arm-leg co-ordination are conserved across different locomotor tasks in human beings (Balter and Zehr, 2007; Patrick et al., 2012), including a reciprocal pattern of influences between the co-ordination of reaching and walking (Chiovetto and Giese, 2013), balance corrective responses (Forero and Misiaszek, 2014), or quadrupedal limb co-ordination during obstacle avoidance (Dietz and Michel, 2009).

\section{GAIT ASSISTED BY EMG ACTIVITY IN UPPER LIMB MUSCLES}

Our study investigated the possibility of extracting spatiotemporal kinematic data from the gait-related rhythmic activity of arm muscles in order to provide a reliable control of walking. The 
results showed that, even with relatively low intensity of upper limb muscle activation, the temporal structure of EMG activity is sufficiently reliable to reproduce the spatiotemporal kinematic pattern of leg movements during walking at constant and variable speed (Figures 2 and 3). A comparison of actual and predicted stride leg kinematics showed a high degree of correspondence $(r>0.9)$. The suggested algorithm has been also implemented in pilot experiments for controlling avatar walking in a virtual reality environment and walking in the exoskeleton (Figure 4). Subjects were able to generate walking kinematics of the avatar for at least $1 \mathrm{~min}$.

Myoelectric signals represent one of the measurable outputs of central nervous system activity. Thus, our study showed that this type of brain-computer interface can be used to link shoulder muscle EMG activity to leg movements (Figures 2 and 3) and to control ambulation within a virtual reality environment (Figure 4A), suggesting that a myoelectric-controlled lower extremity prosthesis for ambulation may be feasible. While the example in Figure 4A involves only four shoulder muscles and the example in Figure 4B involves two muscles, the implemented on-line algorithm can include the sum of bilateral EMGs of synergistic muscles (trapezius, latissimus dorsi, posterior and anterior portions of deltoid muscle, etc., individually adjusted for each patient to provide maximum comfort/efficiency) that are normally active during walking (Ivanenko et al., 2006; Kuhtz-Buschbeck and Jing, 2012). In fact, multi-muscle synergy-based control interface may be more efficient than a single-muscle control (Lunardini et al., 2014), as it also tended to be the case in our experiments when comparing predictions from two EMGs vs. four EMGs (Figure 2D), although further investigations are needed to compare different approaches, especially in neurological injuries with impaired inter-limb co-ordination.

Currently, many research projects are trying to apply novel, physiologically inspired control methods to provide an intuitive way for a patient to command an exoskeleton. Despite its deceiving simplicity, it is worth stressing that the implemented algorithm (Figure 1) takes into account a natural coupling of leg and arm movements during normal walking. Automaticity of arm swinging may be beneficial for the control of rhythmic leg movements, as opposed to a step-by-step voluntary control of muscles that are typically not involved in locomotion (e.g., when using push buttons or finger tapping for triggering stepping of a leg assistive robot that requires continuous cognitive resources, Lisi et al., 2014).

Finally, in addition to gait assistive aspects of exoskeleton robotic devices in severely paralyzed individuals, the proposed approach may also be beneficial for gait rehabilitation in less severe paresis of the lower limbs. Rhythmic upper limb muscle activation has an excitatory effect on lower limb muscle activation during locomotor-like tasks (Ferris et al., 2006; Massaad et al., 2014; Sylos-Labini et al., 2014a). To ensure a high level of EMG activity in deltoid (especially at a lower walking speed), the subjects performed slightly larger arm swinging than they usually do, which may require some additional voluntary control. Nevertheless, a special neural coupling occurs between arms and legs when arms move in alternation (Massaad et al., 2014), as well as active engagement of supraspinal motor areas reinforces CPG circuitry functioning (van den Brand et al., 2012; Solopova et al., 2014). This suggests that locomotor rehabilitation therapy after neurological injury should incorporate simultaneous arm and leg rhythmic exercise to take advantage of neural coupling. Such investigations stimulate new developments of neuroprosthetic technology and provide further insights into how we can integrate biological principles of control with an electromechanical exoskeleton that augments human performance for both gait assistive and rehabilitation technology.

\section{ACKNOWLEDGMENTS}

The authors thank Barbara La Scaleia for the help with using the UDP protocol and software for controlling virtual avatar and Mathieu Duvinage for the help with experiments. This work was supported by the Italian Health Ministry, Italian Ministry of University and Research (PRIN project), Italian Space Agency (CRUSOE and COREA grants), and European Union FP7-ICT program (MINDWALKER grant \#247959). Valentina La Scaleia is supported by the Ph.D. program in Neurosciences of the University of Rome Tor Vergata.

\section{REFERENCES}

Alcaide-Aguirre, R. E., Morgenroth, D. C., and Ferris, D. P. (2013). Motor control and learning with lower-limb myoelectric control in amputees. J. Rehabil. Res. Dev. 50, 687. doi:10.1682/JRRD.2012.06.0115

Ballesteros, M. L., Buchthal, F., and Rosenfalck, P. (1965). The pattern of muscular activity during the arm swing of natural walking. Acta Physiol. Scand. 63 , 296-310. doi:10.1111/j.1748-1716.1965.tb04069.x

Balter, J. E., and Zehr, E. P. (2007). Neural coupling between the arms and legs during rhythmic locomotor-like cycling movement. J. Neurophysiol. 97, 1809-1818. doi:10.1152/jn.01038.2006

Barthelemy, D., and Nielsen, J. B. (2010). Corticospinal contribution to arm muscle activity during human walking. J. Physiol. (Lond.) 588, 967-979. doi:10.1113/ jphysiol.2009.185520

Bianchi, L., Angelini, D., and Lacquaniti, F. (1998). Individual characteristics of human walking mechanics. Pflugers Arch. 436, 343-356. doi:10.1007/ s004240050642

Borghese, N. A., Bianchi, L., and Lacquaniti, F. (1996). Kinematic determinants of human locomotion. J. Physiol. (Lond.) 494(Pt 3), 863-879.

Cappellini, G., Ivanenko, Y. P., Poppele, R. E., and Lacquaniti, F. (2006). Motor patterns in human walking and running. J. Neurophysiol. 95, 3426-3437. doi:10.1152/jn.00081.2006

Cheron, G., Duvinage, M., De Saedeleer, C., Castermans, T., Bengoetxea, A., Petieau, M., et al. (2012). From spinal central pattern generators to cortical network: integrated BCI for walking rehabilitation. Neural Plast. 2012, 375148 doi:10.1155/2012/375148

Chiovetto, E., and Giese, M. A. (2013). Kinematics of the coordination of pointing during locomotion. PLoS ONE 8:e79555. doi:10.1371/journal.pone.0079555

Collins, S. H., Adamczyk, P. G., and Kuo, A. D. (2009). Dynamic arm swinging in human walking. Proc. Biol. Sci. 276, 3679-3688. doi:10.1098/rspb.2009.0664

De Sèze, M., Falgairolle, M., Viel, S., Assaiante, C., and Cazalets, J.-R. (2008). Sequential activation of axial muscles during different forms of rhythmic behavior in man. Exp. Brain Res. 185, 237-247. doi:10.1007/s00221-007-1146-2

del-Ama, A. J., Moreno, J. C., Gil-Agudo, A., de-los-Reyes, A., and Pons, J. L. (2012). Online assessment of human-robot interaction for hybrid control of walking. Sensors 12, 215-225. doi:10.3390/s120100215

Dietz, V., and Michel, J. (2009). Human bipeds use quadrupedal coordination during locomotion. Ann. N. Y. Acad. Sci. 1164, 97-103. doi:10.1111/j.1749-6632.2008. 03710.x

Falgairolle, M., de Seze, M., Juvin, L., Morin, D., and Cazalets, J.-R. (2006). Coordinated network functioning in the spinal cord: an evolutionary perspective. J. Physiol. Paris 100, 304-316. doi:10.1016/j.jphysparis.2007.05.003

Ferris, D. P., Huang, H. J., and Kao, P.-C. (2006). Moving the arms to activate the legs. Exerc. Sport Sci. Rev. 34, 113-120. doi:10.1249/00003677-20060700000005 
Fitzsimmons, N. A., Lebedev, M. A., Peikon, I. D., and Nicolelis, M. A. L. (2009). Extracting kinematic parameters for monkey bipedal walking from cortical neuronal ensemble activity. Front. Integr. Neurosci. 3:3. doi:10.3389/neuro.07.003. 2009

Ford, M. P., Wagenaar, R. C., and Newell, K. M. (2007). Arm constraint and walking in healthy adults. Gait Posture 26, 135-141. doi:10.1016/j.gaitpost.2006.08.008

Forero, J., and Misiaszek, J. E. (2014). Balance corrective responses to unexpected perturbations at the arms during treadmill walking. J. Neurophysiol. 112, 1790-1800. doi:10.1152/jn.00719.2013

Gordon, K. E., Kinnaird, C. R., and Ferris, D. P. (2013). Locomotor adaptation to a soleus EMG-controlled antagonistic exoskeleton. J. Neurophysiol. 109 1804-1814. doi:10.1152/jn.01128.2011

Gwin, J. T., Gramann, K., Makeig, S., and Ferris, D. P. (2011). Electrocortical activity is coupled to gait cycle phase during treadmill walking. Neuroimage 54, 1289-1296. doi:10.1016/j.neuroimage.2010.08.066

Hogue, R. E. (1969). Upper-extremity muscular activity at different cadences and inclines during normal gait. Phys. Ther. 49, 963-972.

Ivanenko, Y. P., Cappellini, G., Poppele, R. E., and Lacquaniti, F. (2008). Spatiotemporal organization of alpha-motoneuron activity in the human spinal cord during different gaits and gait transitions. Eur. J. Neurosci. 27, 3351-3368. doi:10.1111/j.1460-9568.2008.06289.x

Ivanenko, Y. P., Poppele, R. E., and Lacquaniti, F. (2006). Spinal cord maps of spatiotemporal alpha-motoneuron activation in humans walking at different speeds. J. Neurophysiol. 95, 602-618. doi:10.1152/jn.00767.2005

Juvin, L., Gal, J.-P. L., Simmers, J., and Morin, D. (2012). Cervicolumbar coordination in mammalian quadrupedal locomotion: role of spinal thoracic circuitry and limb sensory inputs. J. Neurosci. 32, 953-965. doi:10.1523/JNEUROSCI. 4640- 11.2012

Kang, H. G., and Dingwell, J. B. (2009). Dynamics and stability of muscle activations during walking in healthy young and older adults. J. Biomech. 42, 2231-2237. doi:10.1016/j.jbiomech.2009.06.038

Kuhtz-Buschbeck, J. P., and Jing, B. (2012). Activity of upper limb muscles during human walking. J. Electromyogr. Kinesiol. 22, 199-206. doi:10.1016/j.jelekin. 2011.08.014

Lacquaniti, F., Ivanenko, Y. P., and Zago, M. (2002). Kinematic control of walking. Arch. Ital. Biol. 140, 263-272.

Lebedev, M. A., Tate, A. J., Hanson, T. L., Li, Z., O’Doherty, J. E., Winans, J. A., et al. (2011). Future developments in brain-machine interface research. Clinics (Sao Paulo) 66(Suppl. 1), 25-32. doi:10.1590/S1807-59322011001300004

Lisi, G., Noda, T., and Morimoto, J. (2014). Decoding the ERD/ERS: influence of afferent input induced by a leg assistive robot. Front. Syst. Neurosci. 8:85. doi:10.3389/fnsys.2014.00085

Lunardini, F., Casellato, C., Berger, D., Bertucco, M., d'Avella, A., Sanger, T., et al. (2014). Multi-muscle synergy-based control of a robotic device: a promising approach for helping people with movement disorders. Neural Control of Movement Society Annual Meeting, Amsterdam.

MacLellan, M. J., Ivanenko, Y. P., Cappellini, G., Sylos Labini, F., and Lacquaniti, F. (2012). Features of hand-foot crawling behavior in human adults. J. Neurophysiol. 107, 114-125. doi:10.1152/jn.00693.2011

Malcolm, P., Derave, W., Galle, S., and De Clercq, D. (2013). A simple exoskeleton that assists plantarflexion can reduce the metabolic cost of human walking. PLoS ONE 8:e56137. doi:10.1371/journal.pone.0056137

Massaad, F., Levin, O., Meyns, P., Drijkoningen, D., Swinnen, S. P., and Duysens, J. (2014). Arm sway holds sway: locomotor-like modulation of leg reflexes when arms swing in alternation. Neuroscience 258, 34-46. doi:10.1016/j.neuroscience. 2013.10.007

Meyns, P., Bruijn, S. M., and Duysens, J. (2013). The how and why of arm swing during human walking. Gait Posture 38, 555-562. doi:10.1016/j.gaitpost. 2013.02.006

Millán, J. D. R., Rupp, R., Müller-Putz, G. R., Murray-Smith, R., Giugliemma, C., Tangermann, M., et al. (2010). Combining brain-computer interfaces and assistive technologies: state-of-the-art and challenges. Front. Neurosci. 4:161. doi:10.3389/fnins.2010.00161

Murray, M. P., Sepic, S. B., and Barnard, E. J. (1967). Patterns of sagittal rotation of the upper limbs in walking. Phys. Ther. 47, 272-284.
Ortega, J. D., Fehlman, L. A., and Farley, C. T. (2008). Effects of aging and arm swing on the metabolic cost of stability in human walking. J. Biomech. 41, 3303-3308. doi:10.1016/j.jbiomech.2008.06.039

Park, J. (2008). Synthesis of natural arm swing motion in human bipedal walking. J. Biomech. 41, 1417-1426. doi:10.1016/j.jbiomech.2008.02.031

Patrick, S. K., Noah, J. A., and Yang, J. F. (2009). Interlimb coordination in human crawling reveals similarities in development and neural control with quadrupeds. J. Neurophysiol. 101, 603-613. doi:10.1152/jn.91125.2008

Patrick, S. K., Noah, J. A., and Yang, J. F. (2012). Developmental constraints of quadrupedal coordination across crawling styles in human infants. J. Neurophysiol. 107, 3050-3061. doi:10.1152/jn.00029.2012

Sale, P., Franceschini, M., Waldner, A., and Hesse, S. (2012). Use of the robot assisted gait therapy in rehabilitation of patients with stroke and spinal cord injury. Eur. J. Phys. Rehabil. Med. 48, 111-121.

Solopova, I. A., Selionov, V. A., Kazennikov, O. V., and Ivanenko, Y. P. (2014). Effects of transcranial magnetic stimulation during voluntary and non-voluntary stepping movements in humans. Neurosci. Lett. 579, 64-69. doi:10.1016/j.neulet. 2014.07.015

Sylos-Labini, F., Ivanenko, Y. P., Maclellan, M. J., Cappellini, G., Poppele, R. E., and Lacquaniti, F. (2014a). Locomotor-like leg movements evoked by rhythmic arm movements in humans. PLoS ONE 9:e90775. doi:10.1371/journal.pone.0090775

Sylos-Labini, F., La Scaleia, V., d'Avella, A., Pisotta, I., Tamburella, F., Scivoletto, G., et al. (2014b). EMG patterns during assisted walking in the exoskeleton. Front. Hum. Neurosci. 8:423. doi:10.3389/fnhum.2014.00423

van den Brand, R., Heutschi, J., Barraud, Q., DiGiovanna, J., Bartholdi, K., Huerlimann, M., et al. (2012). Restoring voluntary control of locomotion after paralyzing spinal cord injury. Science 336, 1182-1185. doi:10.1126/science.1217416

Wagner, J., Solis-Escalante, T., Grieshofer, P., Neuper, C., Müller-Putz, G., and Scherer, R. (2012). Level of participation in robotic-assisted treadmill walking modulates midline sensorimotor EEG rhythms in able-bodied subjects. Neuroimage 63, 1203-1211. doi:10.1016/j.neuroimage.2012.08.019

Wang, L., Wang, S., van Asseldonk, E. H. F., and van der Kooij, H. (2013). "Actively controlled lateral gait assistance in a lower limb exoskeleton," in 2013 IEEE/RSI International Conference on Intelligent Robots and Systems (IROS), Tokyo, 965-970.

Wannier, T., Bastiaanse, C., Colombo, G., and Dietz, V. (2001). Arm to leg coordination in humans during walking, creeping and swimming activities. Exp. Brain Res. 141, 375-379. doi:10.1007/s002210100875

Webb, D., Tuttle, R. H., and Baksh, M. (1994). Pendular activity of human upper limbs during slow and normal walking. Am. J. Phys. Anthropol. 93, 477-489. doi:10.1002/ajpa.1330930407

Wolpaw, J. R. (2013). Brain-computer interfaces. Handb. Clin. Neurol. 110, 67-74. doi:10.1016/B978-0-444-52901-5.00006-X

Zehr, E. P., and Duysens, J. (2004). Regulation of arm and leg movement during human locomotion. Neuroscientist. 10,347-361. doi:10.1177/1073858404264680

Zelik, K. E., La Scaleia, V., Ivanenko, Y. P., and Lacquaniti, F. (2014). Can modular strategies simplify neural control of multidirectional human locomotion? J. Neurophysiol. 111, 1686-1702. doi:10.1152/jn.00776.2013

Conflict of Interest Statement: The authors declare that the research was conducted in the absence of any commercial or financial relationships that could be construed as a potential conflict of interest.

Received: 08 May 2014; accepted: 01 October 2014; published online: 20 October 2014. Citation: La Scaleia V, Sylos-Labini F, Hoellinger T, Wang L, Cheron G, Lacquaniti F and Ivanenko YP (2014) Control of leg movements driven by EMG activity of shoulder muscles. Front. Hum. Neurosci. 8:838. doi: 10.3389/fnhum.2014.00838 This article was submitted to the journal Frontiers in Human Neuroscience. Copyright (C) 2014 La Scaleia, Sylos-Labini, Hoellinger, Wang, Cheron, Lacquaniti and Ivanenko. This is an open-access article distributed under the terms of the Creative Commons Attribution License (CC BY). The use, distribution or reproduction in other forums is permitted, provided the original author(s) or licensor are credited and that the original publication in this journal is cited, in accordance with accepted academic practice. No use, distribution or reproduction is permitted which does not comply with these terms. 